\title{
Prefrontal Functional Connectivity During the Verbal Fluency Task in Patients With Major Depressive Disorder: A Functional Near-Infrared Spectroscopy Study
}

\author{
Suh-Yeon Dong ${ }^{1}$, JongKwan $\mathrm{Choi}^{2}$, Yeonsoo Park ${ }^{3}$, Seung Yeon Baik ${ }^{4}$, Minjee Jung ${ }^{5}$, \\ Yourim Kim $^{6}$ and Seung-Hwan Lee ${ }^{5,7 *}$ \\ ${ }^{1}$ Department of Information Technology Engineering, Sookmyung Women's University, Seoul, South Korea, ${ }^{2}$ OBELAB, \\ Seoul, South Korea, ${ }^{3}$ Department of Psychology, University of Notre Dame, Dame, Rl, United States, ${ }^{4}$ Department of \\ Psychology, Penn State University, State College, PA, United States, ${ }^{5}$ Clinical Emotion and Cognition Research Laboratory, \\ Inje University, Goyang, South Korea, ${ }^{6}$ Department of Psychology, University of Wisconsin-Milwaukee, Milwaukee, WI, \\ United States, ${ }^{7}$ Department of Psychiatry, Inje University Ilsan Paik Hospital, Goyang, South Korea
}

\section{OPEN ACCESS}

Edited by:

Tae Young Lee,

Pusan National University Yangsan

Hospital, South Korea

Reviewed by:

Sung-Hwa Ko,

Pusan National University Yangsan

Hospital, South Korea

Junhee Lee,

Seoul National University Hospital,

South Korea

Wu Jeong Hwang,

Seoul National University, South Korea

${ }^{*}$ Correspondence:

Seung-Hwan Lee

Ishpss@hanmail.net

Specialty section:

This article was submitted to Neuroimaging and Stimulation, a section of the journal

Frontiers in Psychiatry

Received: 29 January 2021 Accepted: 14 April 2021 Published: 21 May 2021

Citation:

Dong S-Y, Choi J, Park Y, Baik SY,

Jung M, Kim Y and Lee S-H (2021)

Prefrontal Functional Connectivity During the Verbal Fluency Task in

Patients With Major Depressive Disorder: A Functional Near-Infrared

Spectroscopy Study.

Front. Psychiatry 12:659814.

doi: 10.3389/fpsyt.2021.659814
Deviations in activation patterns and functional connectivity have been observed in patients with major depressive disorder (MDD) with prefrontal hemodynamics of patients compared with healthy individuals. The graph-theoretical approach provides useful network metrics for evaluating functional connectivity. The evaluation of functional connectivity during a cognitive task can be used to explain the neurocognitive mechanism underlying the cognitive impairments caused by depression. Overall, 31 patients with MDD and 43 healthy individuals completed a verbal fluency task (VFT) while wearing a head-mounted functional near-infrared spectroscopy (fNIRS) devices. Hemodynamics and functional connectivity across eight prefrontal subregions in the two groups were analyzed and compared. We observed a reduction in prefrontal activation and weaker overall and interhemispheric subregion-wise correlations in the patient group compared with corresponding values in the control group. Moreover, efficiency, the network measure related to the effectiveness of information transfer, showed a significant between-group difference [t $(71.64)=3.66$, corrected $p<0.001]$ along with a strong negative correlation with depression severity ( $r h o=-0.30, p=0.009$ ). The patterns of prefrontal functional connectivity differed significantly between the patient and control groups during the VFT. Network measures can quantitatively characterize the reduction in functional connectivity caused by depression. The efficiency of the functional network may play an important role in the understanding of depressive symptoms.

Keywords: major depressive disorder, fNIRS, functional connectivity, verbal fluency task, efficiency

\section{INTRODUCTION}

Impairments in cognitive functions, such as attention, working memory, and executive function, have been reportedly observed in patients with major depressive disorder (MDD). Studies comparing patients and healthy controls have reported that these impairments are associated with abnormal patterns of brain activity. When compared to healthy individuals, patients with MDD have been reported to display aberrant neuropsychological characteristics. Earlier studies have used 
functional magnetic resonance imaging (fMRI) to reveal reduced prefrontal activation during digit-sorting tasks (1) and verbal fluency tasks (VFTs) (2), imbalance between left and right prefrontal activation during emotional judgment (3), and reduced activation in the right nucleus accumbens during the monetary incentive delay task (4).

The association between prefrontal cortex (PFC) activation and depressive symptoms has also been investigated using near-infrared spectroscopy (NIRS). Multichannel NIRS is an emerging neuroimaging tool that in addition to detecting the spatiotemporal characteristics of brain function using a noninvasive, portable, and restraint-free technique (5), has been validated in psychiatry patients (6). Recently, studies using NIRS have reported a reduction in prefrontal hemodynamic activation in patients with MDD during the VFT, along with a strong negative correlation between the severity of depression and changes in oxygenated hemoglobin ( $\mathrm{HbO} 2)$ (7-9). However, the functional connectivity has not yet been investigated in depth. Some recent studies have characterized distinct resting-state MRI based-functional connectivity in patients with MDD (10), apathetic depression (11), and remitted MDD (12). Kawano et al. reported a strong negative correlation between the HAMD-21 score and average $\mathrm{HbO} 2$ concentration in the frontal lobe (9); however, they did not assess the functional connectivity.

Since the human brain consists of a complex network responsible for its function, considerable attention has been given to the graph theory approach for explaining functional deviations in brain organization resulting from neurological disorders such as schizophrenia (13-15), Alzheimer's disease $(16,17)$, bipolar disorder (18), attention-deficit hyperactivity disorder (19), and depression (20, 21). Although a majority of the earlier studies on functional connectivity relied on fMRI and electroencephalography (EEG) (21), functional NIRS (fNIRS) can be considered as a reasonable alternative for detecting changes in brain functional networks, owing to its high temporal resolution. A recent review paper on the application of fNIRS in MDD research provided comprehensive evidence that the functional connectivity measured by fNIRS is potentially useful for assessing MDD (22). Zhu et al. reported that patients with affective disorders exhibit significantly reduced connectivity in the PFC using resting-state fNIRS (23). Rosenbaum et al. conducted a functional connectivity analysis on patients with late-life depression (24) and further examined functional connectivity in the cortical areas of the default mode network (25). However, to the best of our knowledge, no studies have investigated the differences in functional connectivity during the VFT task.

In this study, we sought to perform an fNIRS-based functional connectivity analysis to investigate PFC activation patterns during the VFT. Unlike the aforementioned studies, which mainly focused on resting-state functional connectivity, we hypothesized that the functional connectivity elicited by cognitive stimulation (using the VFT in this case) differs between patients with MDD and healthy individuals, and that these differences can be visually described by the prefrontal functional network. As a decrease in verbal fluency has been primarily found in patients with MDD, functional connectivity during
TABLE 1 | Participant demographic data $(N=74)$.

\begin{tabular}{lccc}
\hline Variable & $\begin{array}{c}\text { Control }(\boldsymbol{N}=\mathbf{4 3}) \\
\text { Mean }(\mathbf{S D})\end{array}$ & $\begin{array}{c}\text { Patient }(\boldsymbol{N}=\mathbf{3 1}) \\
\text { Mean (SD) }\end{array}$ & $\boldsymbol{p}$-value \\
\hline Age (years) & $34.26(12.47)$ & $39.48(13.82)$ & 0.093 \\
HAMD & $2.02(1.99)$ & $23.55(9.47)$ & $<0.001$ \\
HAMA & $1.42(1.67)$ & $22.32(10.78)$ & $<0.001$ \\
BDI-II & $5.33(3.96)$ & $25.48(12.21)$ & $<0.001$ \\
STAl-state & $31.44(6.75)$ & $54.94(11.98)$ & $<0.001$ \\
STAI-trait & $35.49(8.78)$ & $59.58(11.99)$ & $<0.001$ \\
\hline
\end{tabular}

HAMD, hamilton rating scale for depression; HAMA, hamilton rating scale for anxiety; $B D I-I I$, beck depression inventory; STAI, state-trait anxiety inventory.

the VFT may play an important role in characterizing MDD. Moreover, we calculated graph theory-based connectivity metrics to quantitatively characterize functional connectivity in each group. We identified the measures that demonstrated significant between-group differences based on the connectivity metrics.

\section{MATERIALS AND METHODS}

\section{Participants}

We enrolled 43 healthy participants (age: $34.26 \pm 12.47$ years, control group) and 31 patients (age: $39.48 \pm 13.81$ years, patient group) with MDD who were undergoing treatment at the university hospital. The participants in the patient group had already been diagnosed with MDD according to the Diagnostic and Statistical Manual of Mental Disorders, Fifth Edition (26) by a board-certified psychiatrist. The participants' symptoms of depression and anxiety were assessed using the Hamilton Depression Rating Scale (HAMD) (27), Hamilton Anxiety Rating Scale (HAMA) (28), Beck Depression Inventory (BDI-II) (29), and State-Trait Anxiety Inventory (STAI) (30), which was composed of two subscales (STAI-state and STAI-trait, respectively). We excluded patients with an HAMD scores $<8$ and healthy individuals with an HAMD scores $>8$.

Table 1 represent the demographic data for all enrolled participants. All participants were recruited from the Department of Psychiatry, Inje University Ilsan Paik Hospital. The study was conducted in accordance with the Declaration of Helsinki and was approved by the Institutional Review Board of Inje University Ilsan Paik Hospital (2017-10-013). All the participants provided written informed consent prior to undergoing the fNIRS experiment.

\section{NIRS Device}

A head-mounted wireless NIRS system (NIRSIT; OBELAB Inc., Seoul, Korea) was used to obtain $\mathrm{HbO} 2$ values from the prefrontal region of the human brain. The NIRS system consists of 24 lasers sources (780/850 nm; maximum power under $1 \mathrm{~mW}$ ) and 32 photodetectors with multiple source-detector spacing $(1.5,2.12,3.0,3.35 \mathrm{~cm})$ resulting in 204 measurement points at a sampling rate of $8.138 \mathrm{~Hz}$ (31). We used 48 channels created by a $3.0 \mathrm{~cm}$ separation between the source and the detector for further analysis. We aligned the marking point on the front of the device 
to the center between the eyes to ensure that the same brain region was recorded. Before wearing the device, the subject's hair were finger-combed away from the forehead to minimize its influence on the measurements. After fixing the device position from the front, the strap and the Velcro hooks on the back were securely fixed to prevent the device from moving while recording.

\section{Activation Task}

Participants were instructed to perform the VFT while wearing the wireless head-mounted fNIRS device. The VFT is a type of cognitive assessment, in which participants are asked to produce as many words as possible from a category (semantic or phonemic) in a given period of time. In this study, phonemic VFT was conducted. This task is usually employed in psychological or neuropsychological assessments to detect cognitive impairment $(32,33)$. The entire procedure consisted of three blocks; each block had a 30-s initial rest and a 60-s VFT (30-s pre-task and 30-s task). Participants were asked to repeat aloud the four Korean consonants, "g," "n," "d," and "r" during the pre-task. During the task, participants were instructed to produce as many Korean words as possible beginning with a designated consonant (randomly presented among the eight consonants, "g," "n," "d," "r," "m," "b," "s," and "h").

\section{Data Analysis}

The detected light signals were filtered using high and lowpass filters at $0.005 \mathrm{~Hz}$ and $0.1 \mathrm{~Hz}$, respectively, to eliminate cardiovascular artifacts and environmental noise. We rejected poor-quality channels with a signal-to-noise ratio under 30 $\mathrm{dB}$ prior to extraction of the hemodynamic data to prevent misinterpretation. The location of the 48 NIRS channels with color-scaled rejection ratios of each subject group are provided in Supplementary Figure 1. Subsequently, the hemodynamic responses were extracted using the modified Beer-Lambert law (34) and baseline corrected with the last $5 \mathrm{~s}$ of the pre-task as baseline for each block. The average amplitude of the baseline was subtracted. Following baseline correction, we obtained the block averaged responses for each channel. As mentioned in Section 2.2, we only used 48 channels in the prefrontal region. As shown in Figure 1, the 48 channels were grouped into eight subregions: the right and left dorsolateral PFC (labeled R1 and L1); the ventrolateral PFC (R2 and L2); the frontopolar PFC (R3 and L3); and the orbitofrontal cortex (R4 and L4). We obtained averaged hemodynamic responses within each subregion, such that each participant's recording became a N-by-8 matrix wherein $\mathrm{N}$ indicated time-domain sample numbers and 8 indicated the number of subregions.

The hemodynamic parameters obtained were analyzed to evaluate the functional connectivity. Specifically, functional connectivity was measured using the strength of temporal correlation of hemodynamics between every possible pair of the subregions. Subsequently, computed prefrontal correlation coefficients were used to extract network measures to represent the global network characteristics, such as density, clustering coefficient, and efficiency. We applied weight thresholding to the correlation coefficients to quantitatively measure and compare the functional connectivity between the two groups. Correlations below the threshold value were set to 0 and were assumed to be weak and non-significant links across a threshold range of 0 to 0.95 with increments of 0.05 , as they could have been spurious connections. Suprathreshold correlations were set to 1; therefore, we were able to obtain a binary adjacency matrix for each threshold value (35). Subsequently, we calculated the three most commonly used global connectivity measures as functions of the threshold value, namely, density, clustering coefficient, and efficiency. Data processing and analysis were performed using MATLAB 2018b (Mathworks, Inc., Natick, MA) and network measures were extracted using the brain connectivity toolbox, which is built into the MATLAB environment (36).

\section{Statistical Analysis}

We used a two-sample $t$-test to assess between-group differences in age, psychological scores, representative means, and functional connectivity measures. Homogeneity of variance was assessed using Levene's test for the equality of variance in a two-sample $t$-test. The degree of freedom was adjusted if the equality of variance was not assumed based on the results of Levene's test. Functional connectivity analysis was performed by calculating Pearson's correlation coefficients between every pair of the represented mean values for the eight subregions. $P$-values obtained from the statistical tests were corrected using the false discovery rate (FDR) for multiple comparisons (37). Following the FDR correction, $p$-values under 0.05 were considered statistically significant. All statistical analyses were performed using IBM SPSS Statistics 23 (IBM Corp. Armonk, NY).

\section{RESULTS}

\section{Age and Psychological Test Scores}

First of all, the two groups in this study had no significant differences in age [ $\mathrm{t}(72)=-1.701, \mathrm{p}=0.093]$. However, the comparison of the five psychological test scores using twosample $t$-tests revealed statistically significant between-group differences. The HAMD scores in the patient group (mean \pm standard deviation: $23.55 \pm 9.47$ ) were significantly higher than those in the control group $(2.02 \pm 2.00)$ [ $\mathrm{t}(31.93)=-12.46$, corrected $p<0.001]$. Similarly, the HAMA scores in the patient group $(22.32 \pm 10.78)$ were significantly higher than those in the control group $(1.42 \pm 1.67)[\mathrm{t}(31.03)=-10.70$, corrected $p<$ 0.001]. Moreover, the remaining three scores showed statistically significant between-group differences [BDI-II, t $(34.59)=-8.86$, corrected $p<0.001$; STAI-state, $\mathrm{t}(43.66)=-9.85$, corrected $p<$ 0.001 ; STAI-trait, $\mathrm{t}(72)=-9.99$, corrected $p<0.001]$.

\section{Task Performance}

We measured the number of correct words and the reaction time as task performance metrics during each VFT task. Between-group differences were assessed using two-sample $t$ tests. The word counts in the control group $(25.40 \pm 6.34)$ were significantly higher than those in the patient group (19.77 \pm 7.53$)$ [t $(71)=3.48, p=0.001]$. Similarly, reaction times in the control group $(3,257.84 \pm 711.64 \mathrm{msec})$ were significantly lower than those in the patient group $(4,368.26 \pm 1,995.05 \mathrm{msec})$ [ $\mathrm{t}(35.54)$ $=-2.97, p=0.005]$. 


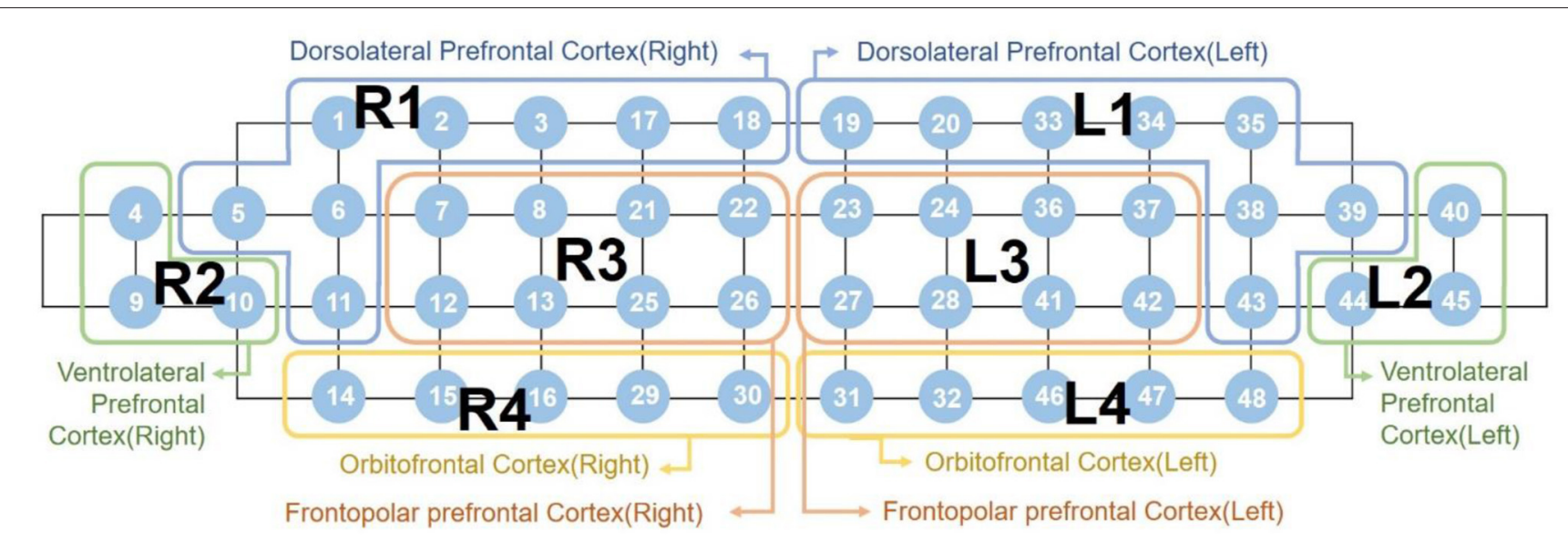

FIGURE 1 | Labeling of the eight subregions mapped by 48 channels "R" indicates the right hemisphere, while "L" indicates the left hemisphere.

\section{fNIRS Activation}

Figure 2 shows the average hemodynamic responses during the VFT in each group. As shown in the figure, the $\mathrm{HbO} 2$ concentration during the VFT was higher in the control group than in the patient group. Figure 3 illustrates the transient hemodynamic responses in each subgroup. The transients of the 48 channels are provided in Supplementary Figure 2. During the task period, the control group showed significantly higher $\mathrm{HbO} 2$ concentrations than the patient group and returned to baseline after the task (not shown in the figure). Moreover, the gap between the two groups was greater in R2, R4, L2, and L4 compared with the other subregions.

\section{Functional Connectivity}

The strength of the temporal correlation of hemodynamics between all pairwise combinations of the eight subgroups was calculated using Pearson's correlation coefficient. As shown in Figure 4A, the strength of the between-subgroup correlation was higher in the control group than in the patient group. The average of the correlation coefficients of the 28 pairs in the control group $(0.68 \pm 0.12)$ was higher than that of the patient group $(0.49 \pm$ $0.16)$. Figure $4 \mathrm{~B}$ shows the spatial distribution of the correlations between each pair of subregions. These figures allow us to observe the areas in which connections are either stronger or weaker. Remarkably, strong interhemispheric connections (e.g., R1-L1, R2-L2, R3-L3, R4-L4) were observed in the control group, but not in the patient group.

We tested the normality of the network measures for each threshold value and evaluated group differences using twosample $t$-tests. First, a significant difference in the clustering coefficient was observed only at the threshold level of 0.90 . The clustering coefficient at 0.9 was significantly larger in the control group $(1.01 \pm 0.25)$ than in the patient group $(0.76 \pm 0.43)[\mathrm{t}$ $(44.522)=2.96$, corrected $p=0.05]$ with a difference of $0.17(95 \%$ CI, 0.082 to 0.43 ). In contrast, density and efficiency exhibited statistically significant between-group differences at all threshold levels $(p<0.05)$. The most significant difference in density was observed at a threshold level of 0.8. At this threshold level, the density in the control group $(0.77 \pm 0.30)$ was significantly higher than that in the patient group $(0.55 \pm 0.21)[\mathrm{t}(72)=$ 3.61 , corrected $p=0.02$ ] with a difference of 0.22 (95\% CI, 0.097 to 0.33 ). As shown in Figure 5A, the most significant difference in efficiency was also found at the threshold level of 0.8. At this threshold level, the efficiency in the control group $(0.56 \pm 0.28)$ was significantly higher than that in the patient group $(0.34 \pm 0.22)[\mathrm{t}(71.64)=3.66$, corrected $\mathrm{p}<0.001]$ with a difference of 0.058 ( $95 \% \mathrm{CI}, 0.097$ to 0.33 ). Moreover, we measured the correlation coefficient between the efficiency at this threshold level and the corresponding HAMD scores. A Spearman's correlation revealed a significant negative correlation between these two factors (rho $=-0.30, p=0.009$ ), as shown in Figure 5B.

\section{DISCUSSION}

In this study, we performed functional connectivity-based comparisons between patients with MDD and healthy individuals and calculated the subregional functional connectivity using temporal correlations of the hemodynamics of every pair of subregions of the PFC using a functional connectivity approach. The functional connectivity in each group (control and patient) was visually represented by varying the thickness according to the strength of the subregional functional connectivity. Network measures were calculated to quantitatively evaluate and compare two networks, and the most informative metric was identified as the network measure representing the greatest difference between the two groups (control vs. patient).

The visualization of functional connectivity allowed us to understand subregion-wise functional connection characteristics between the two groups. We observed a clear absence of interhemispheric correlations in patients with MDD. This result is consistent with a previous study indicating that patients with MDD show interhemispheric connectivity deficits in restingstate (38). 

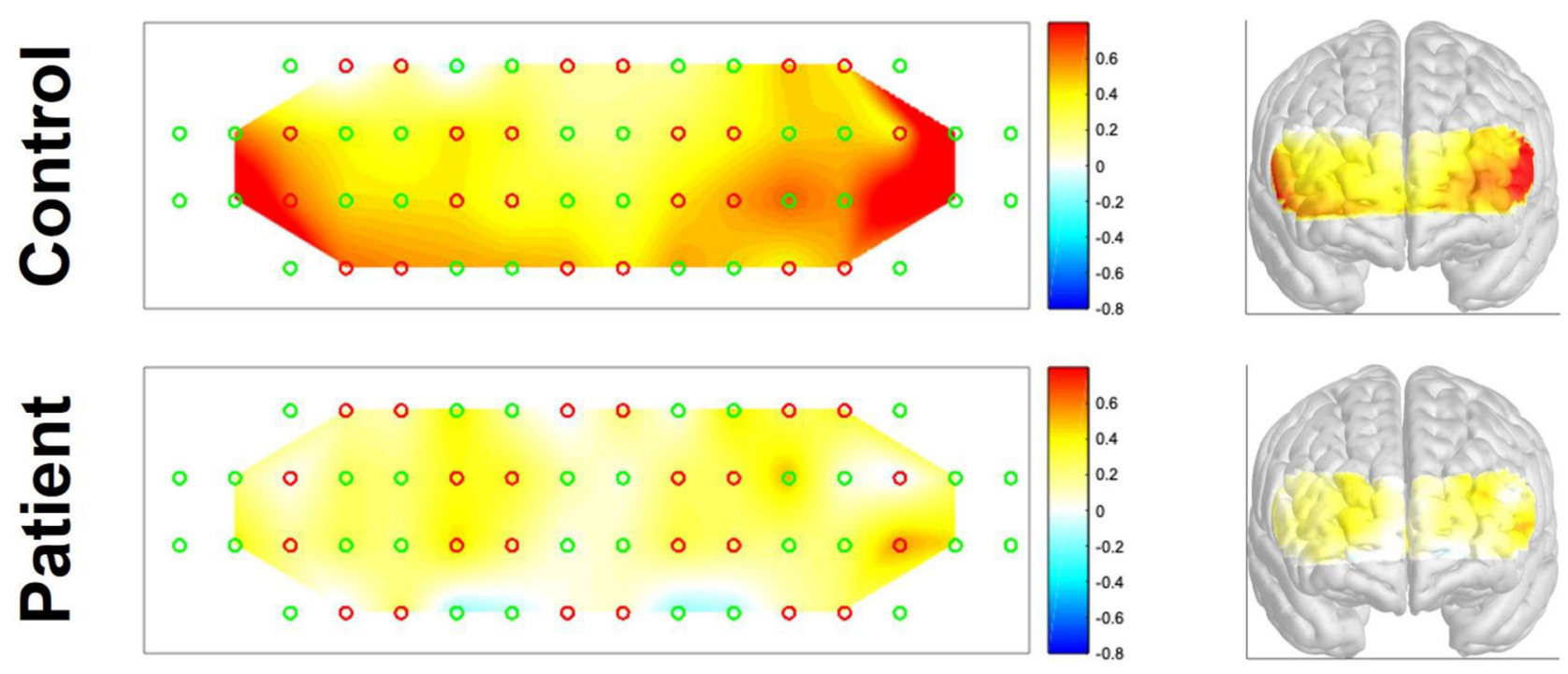

FIGURE 2 | Activation maps of the two groups during the verbal fluency task.

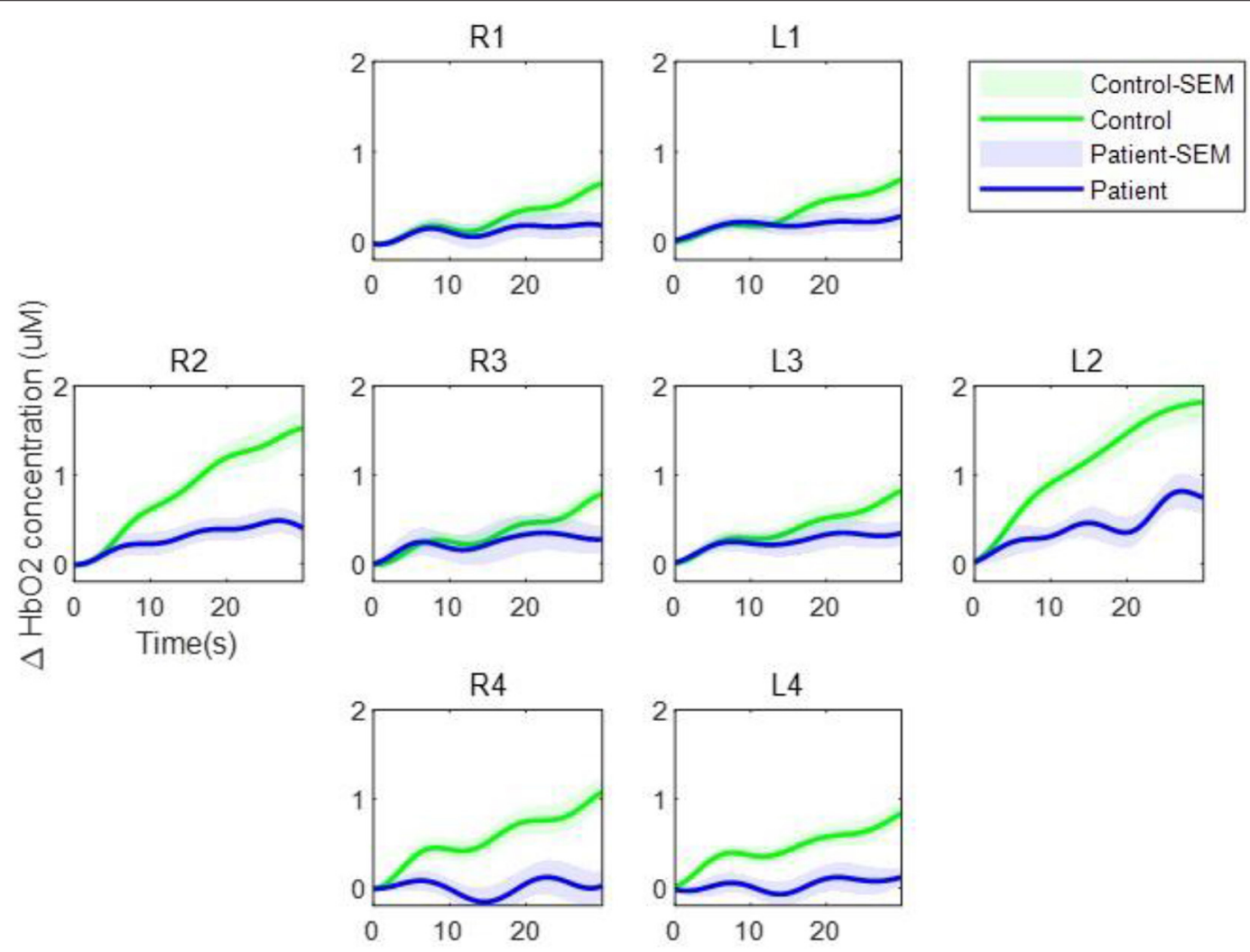

FIGURE 3 | Transient oxygenated hemoglobin concentration during the verbal fluency task. The green line represents the control group while the blue line represents the patient group. The shaded regions represent the standard errors of the mean.

The network measures used in our study are known to characterize basic aspects of network organization (35). Density reflects the overall wiring cost of the network.
The clustering coefficient indicates network segregation, while efficiency reflects the network integration (36). Of the two measures, we focused extensively on efficiency, since it showed 


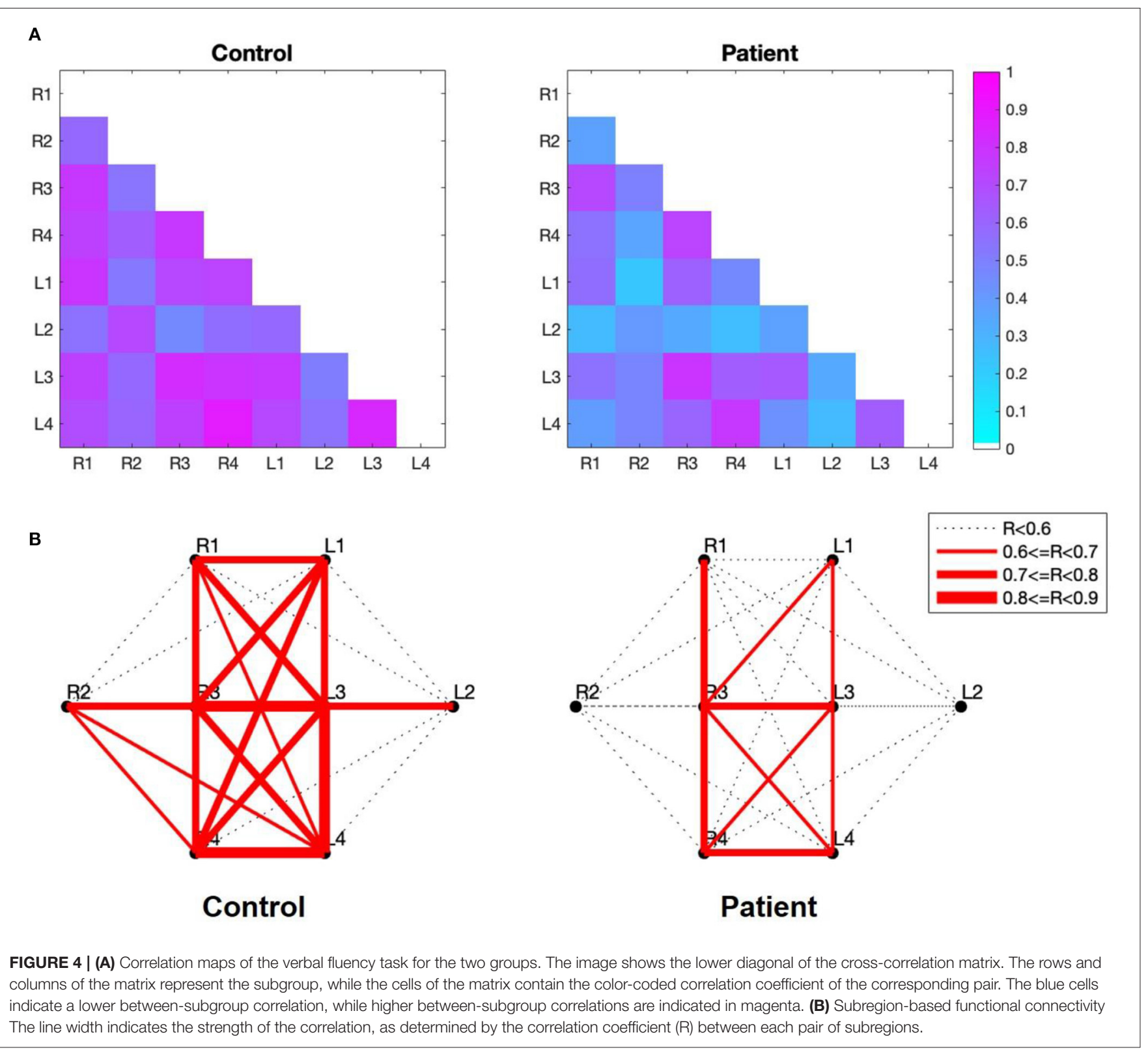

a significant between-group difference. We observed a strong negative correlation between fNIRS measures and the severity of depression, which is in line with other two studies $(8,9)$. Unlike these studies, which calculated correlations based on changes in $\mathrm{HbO} 2$ from a single channel (8) or an entire frontal channel (9), we used the network measure instead of $\mathrm{HbO} 2$ changes in this study; thus, we were able to offernew interpretations of the relationship between fNIRS measurement and depression severity. As efficiency is a measure of the effectiveness of information transfer, efficiency may play an important role in understanding decreased responsiveness in patients with MDD, including a slower reaction time (4). Although the Spearman's rho between reaction time and efficiency was not statistically significant in our study (rho $=-0.21, p=0.073$ ), efficiency was the most negatively correlated network metric of the three. Future studies with a larger population may provide experimental evidence. Thus, the results so far demonstrate that these network metrics may be used as valid biomarkers of MDD.

Since most previous studies have focused primarily on resting-state functional connectivity (23-25), this study is distinct in that it suggests task-related functional connectivity. While Rosenbaum et al. have also investigated the functional connectivity during task performance as well as at rest (24, 25), the task adopted in these studies on late-life depression was a trail-making test that is often used for screening visual attention and task switching. This study uses the VFT adopted in the majority of previous studies of MDD. Therefore, we believe that our findings provide meaningful evidence that functional connectivity may be used to understand the behavioral and neurological characteristics of decreased verbal fluency in patients with MDD. 

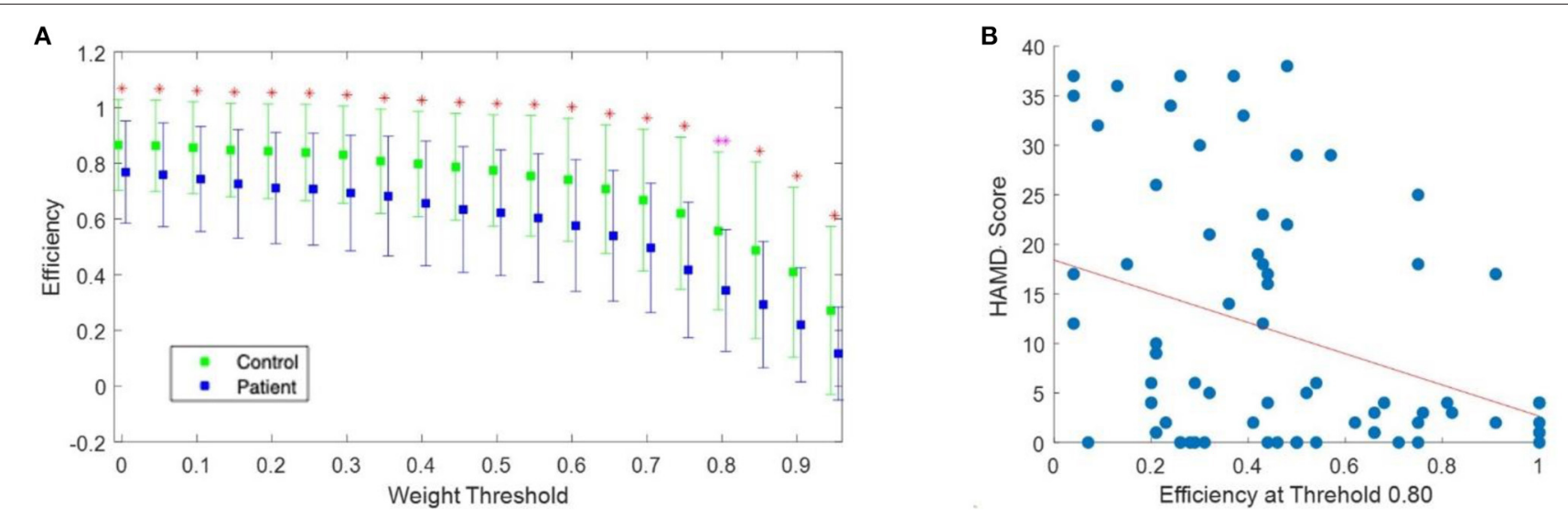

FIGURE 5 | (A) Efficiency as a function of the threshold value. The green squares indicate efficiency in the control group while the blue squares indicate efficiency in the patient group. The error bar indicates the standard deviation. The magenta double asterisk indicates a significant difference at the level of 0.001 , while red asterisks indicate a significant difference at the level of 0.05 ( $p$-values were FDR-corrected). (B) Scatter plot of the efficiency at a threshold of 0.80 vs HAMD scores. The red line indicates the least-squares line. FDR, false discovery rate; HAMD, hamilton depression rating scale.

\section{DATA AVAILABILITY STATEMENT}

The datasets generated for this study cannot be made openly available due to ethical concerns. Requests to access the datasets should be directed to Seung-Hwan Lee, 1shpss@hanmail.net.

\section{ETHICS STATEMENT}

The studies involving human participants were reviewed and approved by Institutional Review Board of Inje University Ilsan Paik Hospital (2017-10-013). The patients/participants provided their written informed consent to participate in this study.

\section{AUTHOR'S NOTE}

This study has some limitations. Given that aging is a critical cause of cognitive decline (17) and in light of the potential need to set clinical cut-off points in rating scales for older patients (39), further investigations on age-related functional connectivity in patients with MDD are necessary. Moreover, although depression severity was not the main focus of our study, stricter criteria for enrolling patients with MDD could have provided more accurate results that better represent the characteristics of MDD.

\section{AUTHOR CONTRIBUTIONS}

S-YD: conceptualization, data analysis, funding acquisition, and writing - original draft. JC: protocol design, data preprocessing, and writing - review \& editing. YP and SB:

\section{REFERENCES}

1. Siegle GJ, Thompson W, Carter CS, Steinhauer SR, Thase ME. Increased amygdala and decreased dorsolateral prefrontal BOLD responses in unipolar protocol design, investigation, and writing - review \& editing. MJ and YK: data collection, writing - review \& editing. S-HL: conceptualization, supervision and writing - review \& editing. All authors contributed to the article and approved the submitted version.

\section{FUNDING}

This research was supported by the Korea Medical Device Development Fund grant funded by the Korea government (the Ministry of Science and ICT, the Ministry of Trade, Industry and Energy, the Ministry of Health \& Welfare, Republic of Korea, the Ministry of Food and Drug Safety) (Grant no. 202013B10), the National Research Foundation of Korea grants (2019R1F1A1058011 and BK21 FOUR), and a research grant from Sookmyung Women's University Research Grants (1-1903-2005).

\section{ACKNOWLEDGMENTS}

We would like to thank all study participants. We would also like to thank Ms. Jiyoung Baek of OBELAB for organizing the data for subsequent analysis.

\section{SUPPLEMENTARY MATERIAL}

The Supplementary Material for this article can be found online at: https://www.frontiersin.org/articles/10.3389/fpsyt. 2021.659814/full\#supplementary-material

depression: related and independent features. Biol Psychiatry. (2007) 61:198209. doi: 10.1016/j.biopsych.2006.05.048

2. Okada G, Okamoto Y, Yamashita H, Ueda K, Takami H, Yamawaki S. Attenuated prefrontal activation during a verbal fluency task in 
remitted major depression. Psychiatry Clin Neurosci. (2009) 63:4235. doi: 10.1111/j.1440-1819.2009.01952.x

3. Grimm S, Beck J, Schuepbach D, Hell D, Boesiger P, Bermpohl F, et al. Imbalance between left and right dorsolateral prefrontal cortex in major depression is linked to negative emotional judgment: an fMRI study in severe major depressive disorder. Biol Psychiatry. (2008) 63:36976. doi: 10.1016/j.biopsych.2007.05.033

4. Carl H, Walsh E, Eisenlohr-Moul T, Minkel J, Crowther A, Moore T, et al. Sustained anterior cingulate cortex activation during reward processing predicts response to psychotherapy in major depressive disorder. J Affect Disord. (2016) 203:204-12. doi: 10.1016/j.jad.2016. 06.005

5. Scholkmann F, Kleiser S, Metz AJ, Zimmermann R, Mata Pavia J, Wolf $U$, et al. A review on continuous wave functional nearinfrared spectroscopy and imaging instrumentation and methodology. Neuroimage. (2014) 85:6-27. doi: 10.1016/j.neuroimage.2013. 05.004

6. Lee J, Folley BS, Gore J, Park S. Origins of spatial working memory deficits in schizophrenia: an event-related fMRI and near-infrared spectroscopy study. PLoS ONE. (2008) 3:e1760. doi: 10.1371/journal.pone.00 01760

7. Pu S, Matsumura H, Yamada T, Ikezawa S, Mitani H, Adachi $A$, et al. Reduced frontopolar activation during verbal fluency task associated with poor social functioning in late-onset major depression: multi-channel near-infrared spectroscopy study. Psychiatry Clin Neurosci. (2008) 62:728-37. doi: 10.1111/j.1440-1819.2008. 01882.x

8. Noda T, Yoshida S, Matsuda T, Okamoto N, Sakamoto $\mathrm{K}$, Koseki S, et al. Frontal and right temporal activations correlate negatively with depression severity during verbal fluency task: a multi-channel near-infrared spectroscopy study. J Psychiatr Res. (2012) 46:905-12. doi: 10.1016/j.jpsychires.2012. 04.001

9. Kawano M, Kanazawa T, Kikuyama H, Tsutsumi A, Kinoshita S, Kawabata Y, et al. Correlation between frontal lobe oxyhemoglobin and severity of depression assessed using near-infrared spectroscopy. J Affect Disord. (2016) 205:154-8. doi: 10.1016/j.jad.2016. 07.013

10. Wu $\mathrm{X}$, Lin $\mathrm{P}$, Yang J, Song $\mathrm{H}$, Yang $\mathrm{R}$, Yang J. Dysfunction of the cingulo-opercular network in first-episode medication-naive patients with major depressive disorder. J Affect Disord. (2016) 200:275-83. doi: 10.1016/j.jad.2016.04.046

11. Alexopoulos GS, Hoptman MJ, Yuen G, Kanellopoulos D, Seirup JK, Lim KO, et al. Functional connectivity in apathy of late-life depression: a preliminary study. J Affect Disord. (2013) 149:398-405. doi: 10.1016/j.jad.2012. 11.023

12. DelDonno SR, Jenkins LM, Crane NA, Nusslock R, Ryan KA, Shankman SA, et al. Affective traits and history of depression are related to ventral striatum connectivity. J Affect Disord. (2017) 221:72-80. doi: 10.1016/j.jad.2017. 06.014

13. Rubinov M, Knock SA, Stam CJ, Micheloyannis S, Harris AWF, Williams LM, et al. Small-world properties of nonlinear brain activity in schizophrenia. Hum Brain Mapp. (2009) 30:403-16. doi: 10.1002/hbm. 20517

14. Damaraju E, Allen EA, Belger A, Ford JM, McEwen S, Mathalon DH, et al. Dynamic functional connectivity analysis reveals transient states of dysconnectivity in schizophrenia. NeuroImage Clin. (2014) 5:298308. doi: 10.1016/j.nicl.2014.07.003

15. Noda T, Nakagome K, Setoyama S, Matsushima E. Working memory and prefrontal/temporal hemodynamic responses during post-task period in patients with schizophrenia: a multi-channel near-infrared spectroscopy study. J Psychiatr Res. (2017) 95:288-98. doi: 10.1016/j.jpsychires.2017. 09.001

16. Tijms BM, Wink AM, de Haan W, van der Flier WM, Stam CJ, Scheltens P, et al. Alzheimer's disease: connecting findings from graph theoretical studies of brain networks. Neurobiol Aging. (2013) 34:202336. doi: 10.1016/j.neurobiolaging.2013.02.020
17. Lin Q, Rosenberg MD, Yoo K, Hsu TW, O’Connell TP, Chun MM. Resting-state functional connectivity predicts cognitive impairment related to Alzheimer's disease. Front Aging Neurosci. (2018) 10:94. doi: 10.3389/fnagi.2018.00094

18. Hirose T, Tsujii N, Mikawa W, Shirakawa O. Delayed hemodynamic responses associated with a history of suicide attempts in bipolar disorder: a multichannel near-infrared spectroscopy study. Psychiatry Res Neuroimaging. (2018) 280:15-21. doi: 10.1016/j.pscychresns.2018. 08.003

19. Ueda S, Ota T, Iida J, Yamamuro K, Yoshino H, Kishimoto N, et al. Reduced prefrontal hemodynamic response in adult attention-deficit hyperactivity disorder as measured by near-infrared spectroscopy. Psychiatry Clin Neurosci. (2018) 72:380-90. doi: 10.1111/pcn.12643

20. He H, Yu Q, Du Y, Vergara V, Victor TA, Drevets WC, et al. Restingstate functional network connectivity in prefrontal regions differs between unmedicated patients with bipolar and major depressive disorders. J Affect Disord. (2016) 190:483-93. doi: 10.1016/j.jad.2015. 10.042

21. Helm K, Viol K, Weiger TM, Tass PA, Grefkes C, Del Monte D, et al. Neuronal connectivity in major depressive disorder: a systematic review. Neuropsychiatr Dis Treat. (2018) 14:2715-37. doi: 10.2147/NDT.S1 70989

22. Ho CSH, Lim LJH, Lim AQ, Chan NHC, Tan RS, Lee SH, et al. Diagnostic and predictive applications of functional near-infrared spectroscopy for major depressive disorder: a Systematic review. Front Psychiatry. (2020) 11:378. doi: 10.3389/fpsyt.2020.00378

23. Zhu H, Xu J, Li J, Peng H, Cai T, Li X, et al. Decreased functional connectivity and disrupted neural network in the prefrontal cortex of affective disorders: a resting-state fNIRS study. J Affect Disord. (2017) 221:13244. doi: 10.1016/j.jad.2017.06.024

24. Rosenbaum D, Hagen K, Deppermann S, Kroczek AM, Haeussinger FB, Heinzel S, et al. State-dependent altered connectivity in late-life depression: a functional near-infrared spectroscopy study. Neurobiol Aging. (2016) 39:5768. doi: 10.1016/j.neurobiolaging.2015.11.022

25. Rosenbaum D, Haipt A, Fuhr K, Haeussinger FB, Metzger FG, Nuerk $\mathrm{HC}$, et al. Aberrant functional connectivity in depression as an index of state and trait rumination. Sci Rep. (2017) 7:2174. doi: 10.1038/s41598-01702277-z

26. American Psychiatric Association. Diagnostic and Statistical Manual of Mental Disorders (DSM-5®). 5th ed. St. Arlington, VA: American Psychiatric Publishing (2013).

27. Hamilton M. A rating scale for depression. J Neurol Neurosurg Psychiatry. (1960) 23:56-62. doi: 10.1136/jnnp.23.1.56

28. Hamilton M. The assessment of anxiety states by rating. Br J Med Psychol. (1959) 32:50-55. doi: 10.1111/j.2044-8341.1959.tb00467.x

29. Beck AT, Steer RA, Brown G. BDI-II, Beck Depression Inventory: Manual. San Antonio: TX: Psychological Corporation (1996).

30. Spielberger CD, Gorsuch R, Lushene RE, Vagg PR, Jacobs GA. Manual for the State-Trait Anxiety Inventory. Palo Alto, CA: Consulting Psychologists Press (1983).

31. Choi JK, Kim JM, Hwang G, Yang J, Choi MG, Bae HM. Time-divided spread-spectrum code-based $400 \mathrm{fW}$-detectable multichannel fNIRS IC for portable functional brain imaging. IEEE J Solid State Circuits. (2016) 51:48495. doi: 10.1109/JSSC.2015.2504412

32. Takizawa R, Fukuda M, Kawasaki S, Kasai K, Mimura M, Pu S, et al. Neuroimaging-aided differential diagnosis of the depressive state. Neuroimage. (2014) 85:498-507. doi: 10.1016/j.neuroimage.2013.05.126

33. Curtis VA, Dixon TA, Bullmore ET, Morris RG, Brammer MJ, Williams SCR, et al. Differential frontal activation in schizophrenic and bipolar patients during verbal fluency. Neuroimage. (1999) 9:111-21. doi: 10.1016/S0165-0327(00)00240-8

34. Delpy DT, Cope M, Van Der Zee P, Arridge S, Wray S, Wyatt J. Estimation of optical pathlength through tissue from direct time of flight measurement. Phys Med Biol. (1988) 33:1433-42. doi: 10.1088/0031-9155/33/12/008

35. Racz FS, Mukli P, Nagy Z, Eke A. Increased prefrontal cortex connectivity during cognitive challenge assessed by fNIRS imaging. Biomed Opt Express. (2017) 8:3842-55. doi: 10.1364/BOE.8.003842 
36. Rubinov M, Sporns O. Complex network measures of brain connectivity: uses and interpretations. Neuroimage (2010) 52:1059-69. doi: 10.1016/j.neuroimage.2009.10.003

37. Singh AK, Dan I. Exploring the false discovery rate in multichannel NIRS. Neuroimage. (2006) 33:542-9. doi: 10.1016/j.neuroimage.2006. 06.047

38. Wang L, Li K, Zhang QE, Zeng YW, Jin Z, Dai WJ, et al. Interhemispheric functional connectivity and its relationships with clinical characteristics in major depressive disorder: A resting state fMRI study. PLoS ONE. (2013) 8:e60191. doi: 10.1371/journal.pone.0 060191

39. Kogan ES, Kabacoff RI, Hersen M, Van Hasselt VB. Clinical cutoffs for the beck depression inventory and the geriatric depression scale with older adult psychiatric outpatients. J Psychopathol Behav Assess. (1994) 16:23342. doi: 10.1007/BF02229210
Conflict of Interest: JC is the inventor of continuous-wave near infrared spectroscopy technology, licensed to KAIST's spin-off company OBELAB, which focuses on non-invasive, optical brain imaging.

The remaining authors declare that the research was conducted in the absence of any commercial or financial relationships that could be construed as potential conflicts of interest.

Copyright (c) 2021 Dong, Choi, Park, Baik, Jung, Kim and Lee. This is an open-access article distributed under the terms of the Creative Commons Attribution License (CC $B Y)$. The use, distribution or reproduction in other forums is permitted, provided the original author(s) and the copyright owner(s) are credited and that the original publication in this journal is cited, in accordance with accepted academic practice. No use, distribution or reproduction is permitted which does not comply with these terms. 